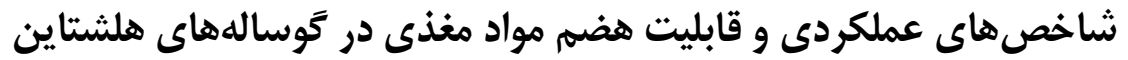

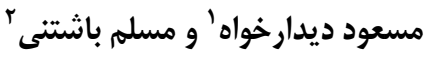

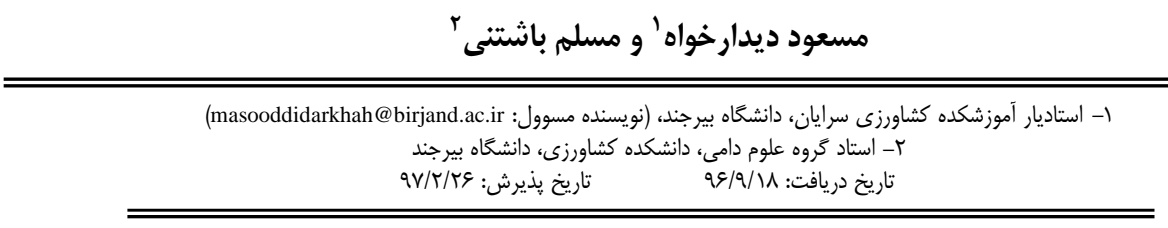

جكيده

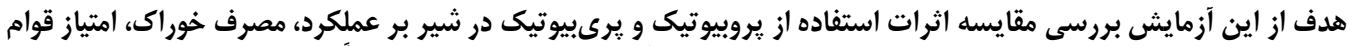

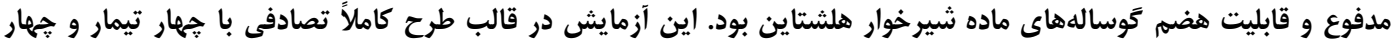

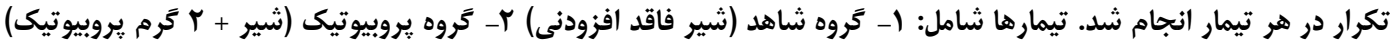

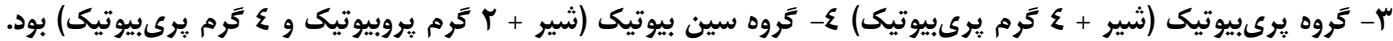

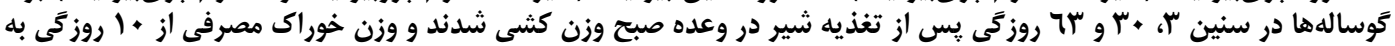

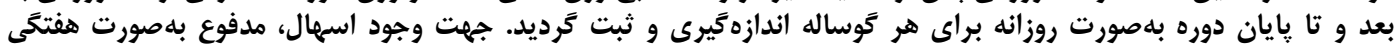

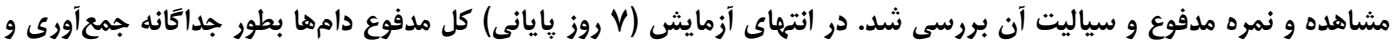

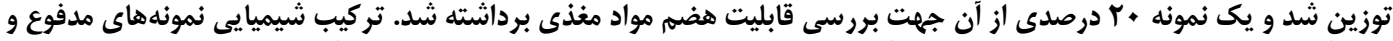

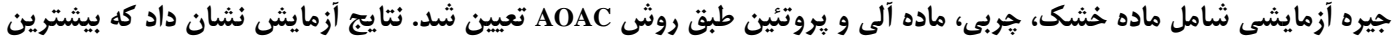

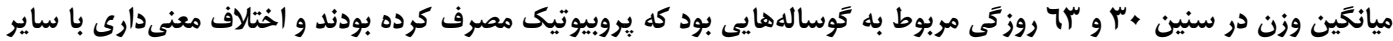

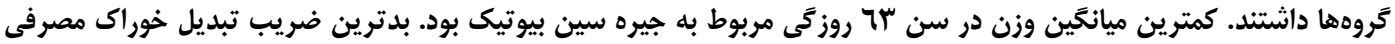

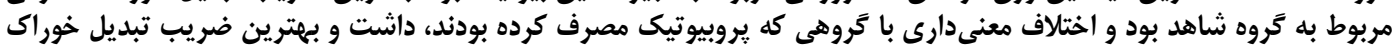

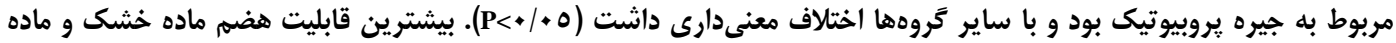

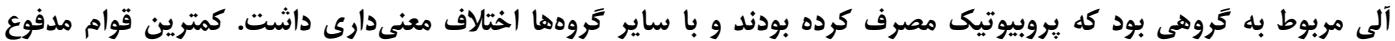

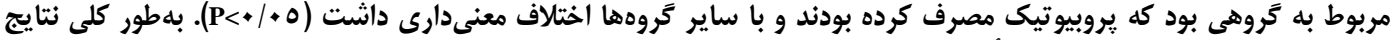

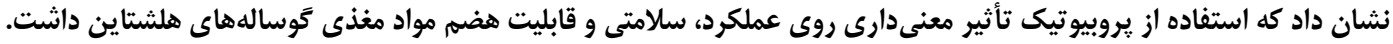

وازههاى كليدى: بروبيوتيك، يرىبيوتيك، نشخوار كنندكان

شده است ولى بسيارى از بررسىها نتايج متغيرى را نشان

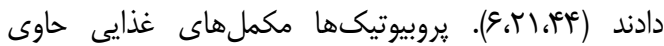

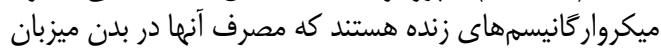

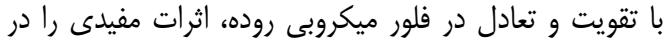

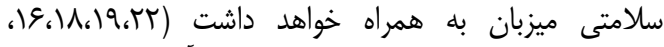

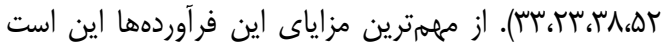

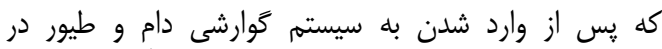

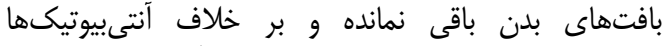

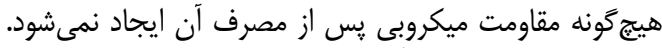

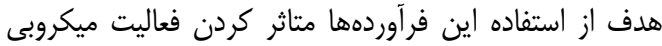

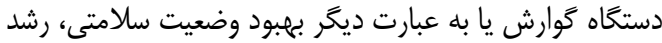

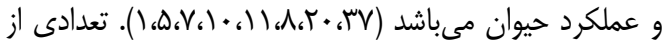

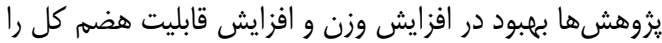

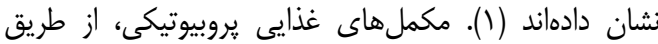

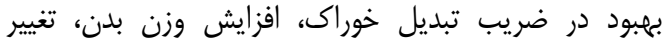

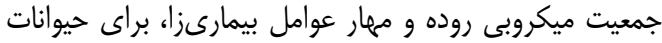

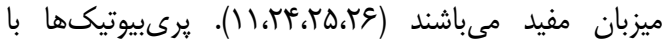

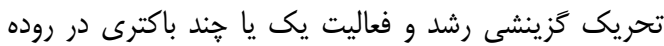

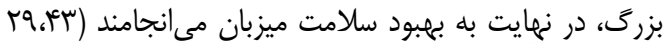

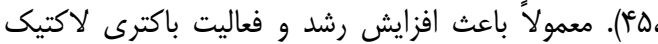

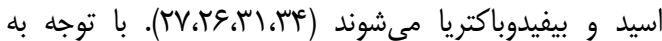
مطالب بحث شده هدف از انجام اين يزوهش براني
مقدمه

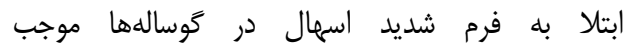

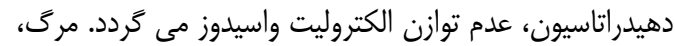

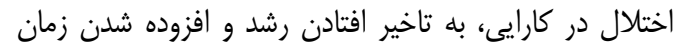

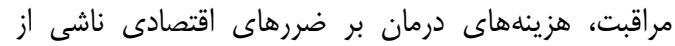

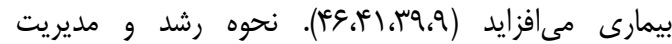

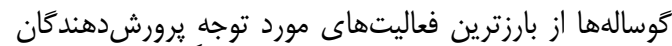

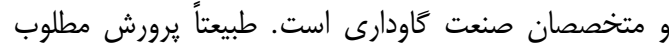

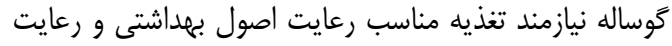

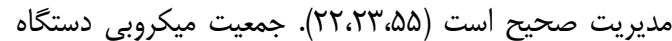

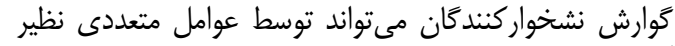

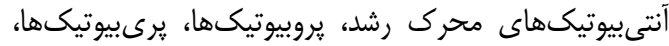

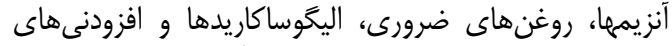

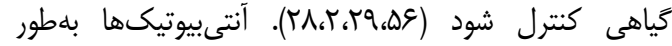

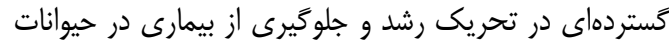

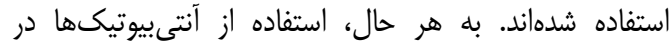

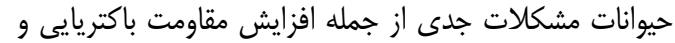

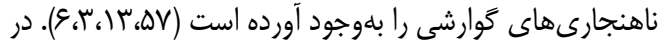

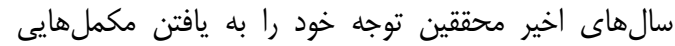

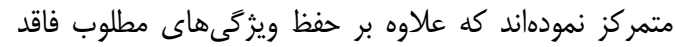

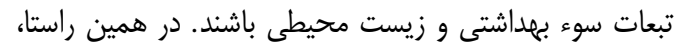
استفاده از يروبيوتيكها و برى يبيوتيكها در كَوسالهها متداول 


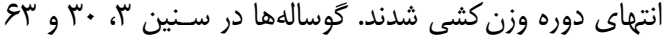

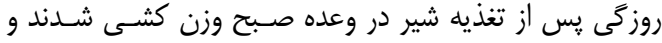

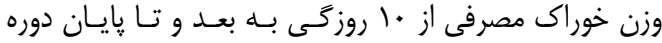

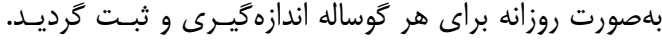

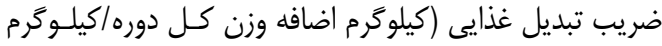

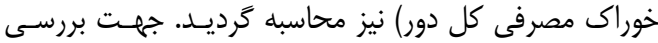

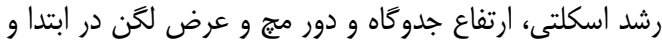

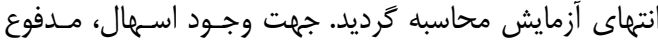

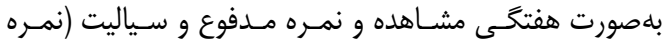

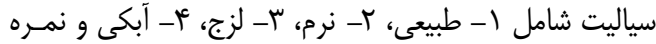

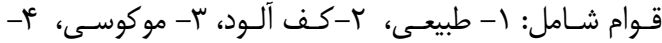

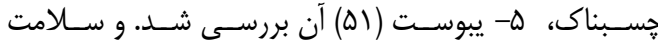

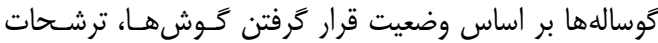

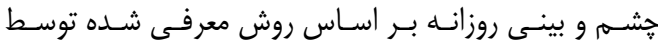

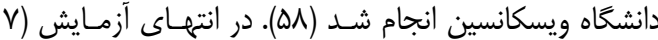

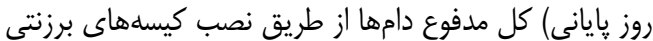

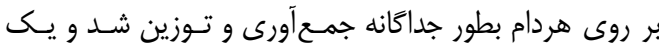

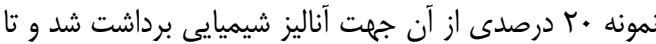

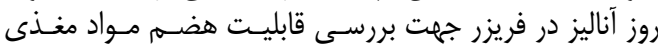

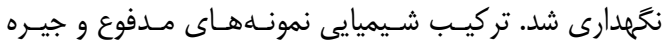

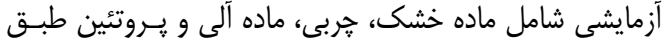
روش AOAC (Tس) تعيين شد. تجزيه و تحليل آمارى

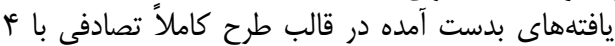

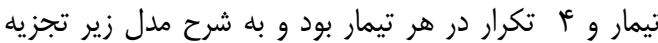

$\mathrm{Yij}=\mu+\mathrm{Ti}+\varepsilon \mathrm{ij}$ كه در آن Yij: مقدار هر مشاهده، بر: اثر ميانخين جامعه،

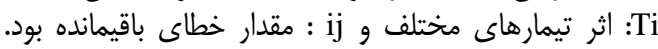

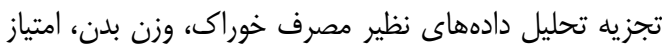

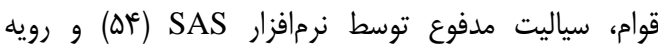

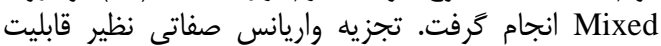
هضم ظاهرى مواد مغذى و ماده خشك توسط نرماف وافزار

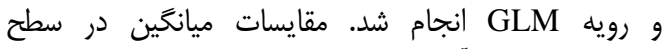

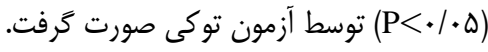

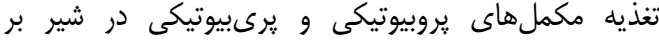

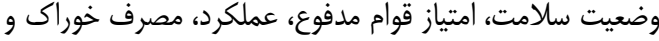

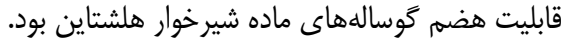

\section{مواد و روشها}

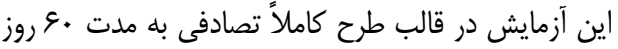

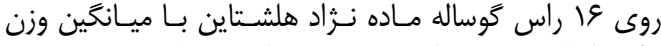

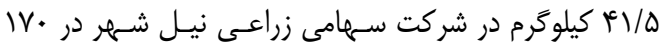

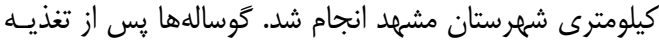

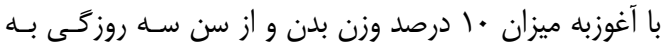

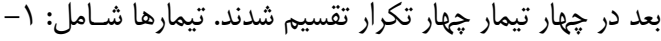

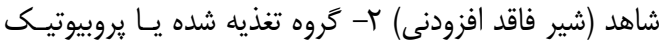

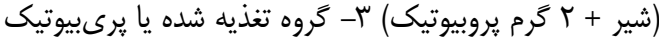

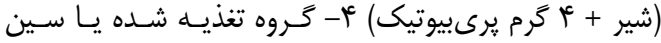

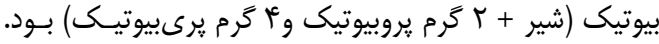

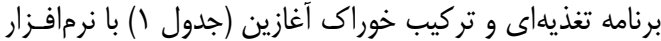
NRC 2001

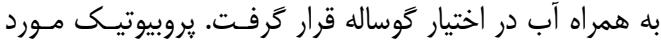

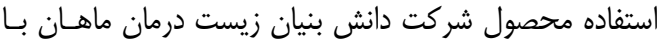

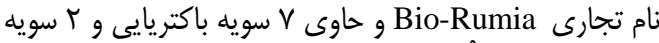

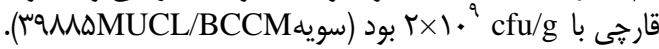

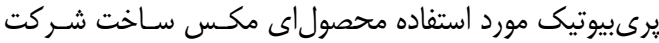
وايكور آمريكا حاوى مخمرساكارومايسس سرويسيـيه و محسيط

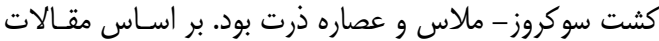

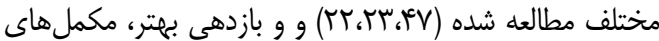

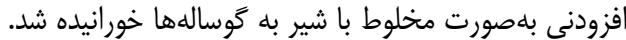

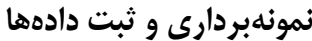

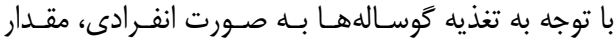

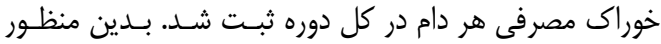

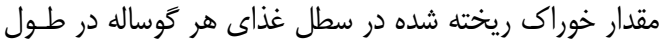

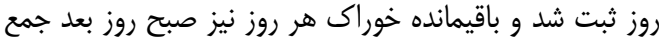

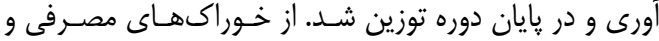

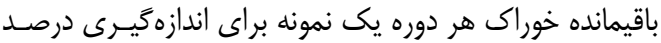

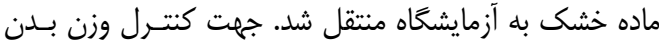

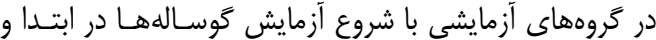

جدول ا- اقلام مواد خوراكى و تركيب شيميايى جيره اغازين كوسالههاى هلشتاين Table 1. The ingredient and chemical composition of the initial diet of Holstein calves

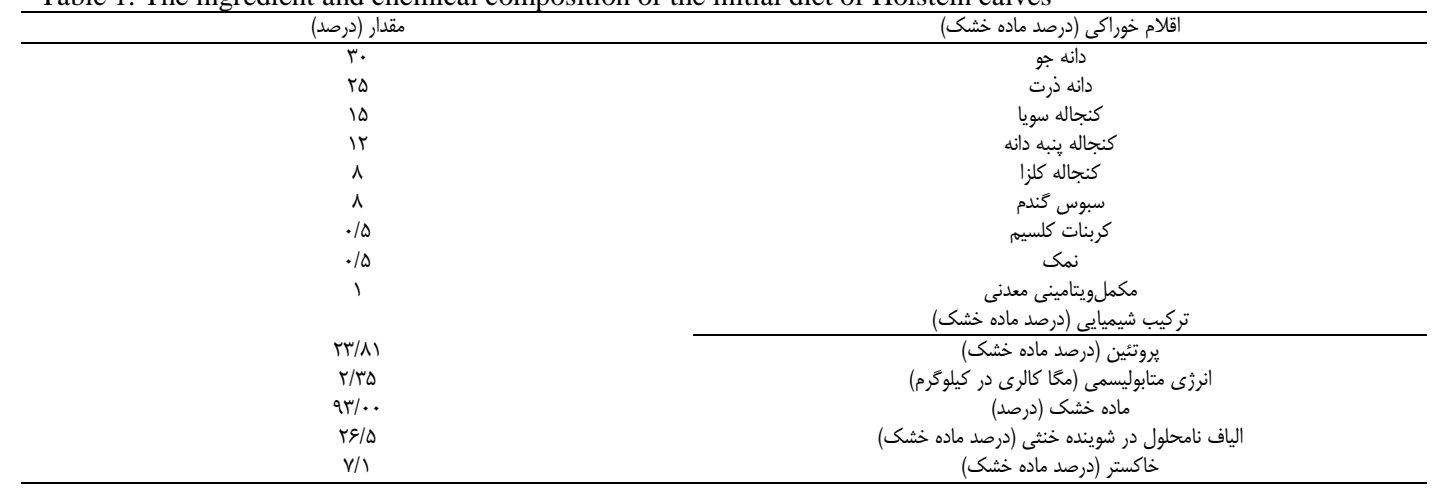



بررسىاثرات استفاده ازافزودنى يروبيوتيك و يرىبيوتيك در شير بر شاخصهاى عملكردى و قابليت هضم مواد مغذى

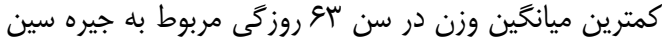

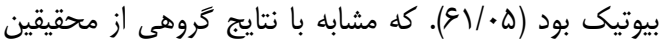

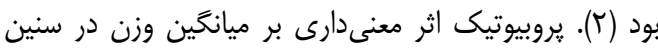

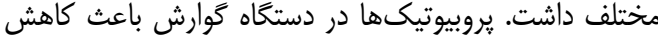

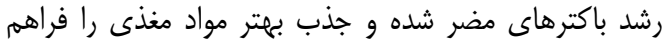

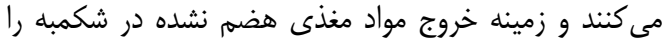

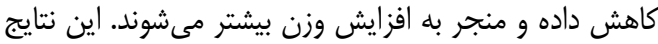

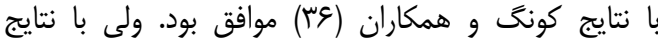

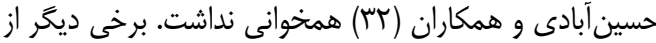

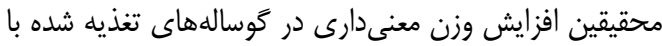

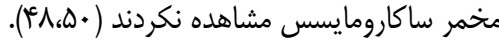

نتايج و بحث

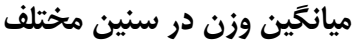

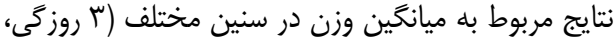

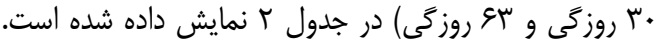

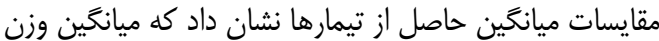

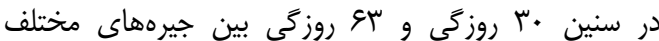

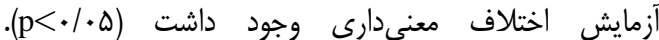

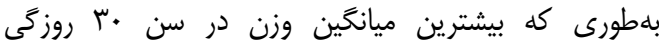
كرده به/DD)

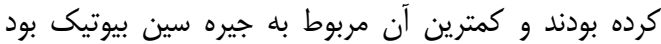
(

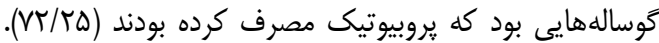

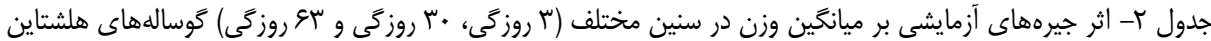
Table 2. Effects of experimental diets on average weight of different age (3 days, 30 days and 63 days) of Holstein calves

\begin{tabular}{|c|c|c|c|c|c|c|}
\hline سطح احتمال & خطاى استانداردميانغين & سين بيوتيك & ي برى بيوتيكى & 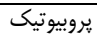 & شاهد & صفات \\
\hline$\cdot / 9$. & $9 / F I T$ & $4 \cdot 1 \cdot 1$ & FI/QT & $F T / Q I$ & $F / / \Delta$. & ميانكَين وزن ״ روزَى (كيلوكَرم) \\
\hline $.1 .+11$ & $1 \% / 9 V T$ & $\varphi g / \Delta r^{D}$ & $r q / \Delta T^{D}$ & $\Delta \Delta / \Delta \omega^{\mathrm{a}}$ & $\Delta \cdot / V \Delta^{\mathrm{ab}}$ & 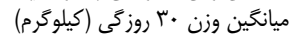 \\
\hline$\cdot / \cdots 1$ & S1. TQ & $81 / \cdot 0^{\mathrm{D}}$ & $S I / T \Delta^{D}$ & $V T / T \Delta^{a}$ & $91 / \mathrm{V} \Delta^{\mathrm{D}}$ & ميانكين وزن سو روزگى (كيلوكرم) \\
\hline
\end{tabular}

افزايش معنى دار ماده خشك مصرفى و بهبود ميانخين افزايش

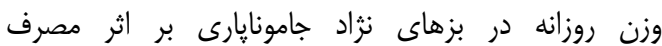

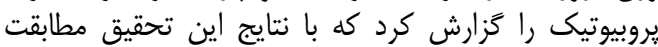

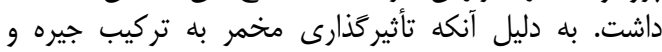

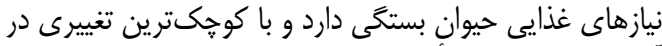

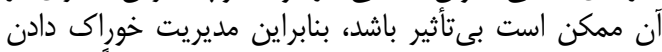

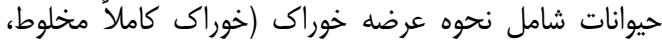

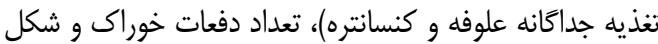

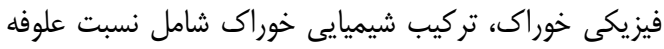

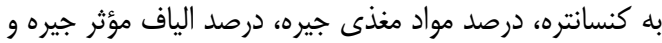

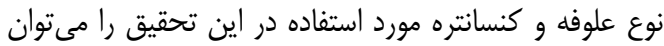

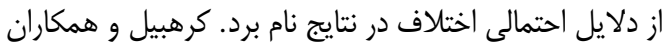

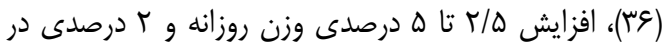

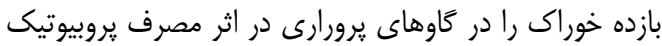

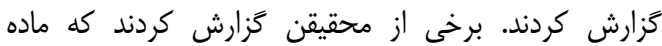

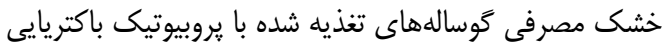

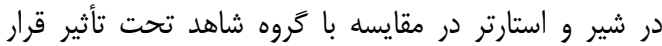

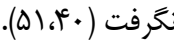

شاخص هاى عملكردى

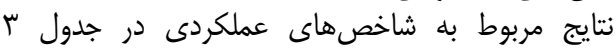

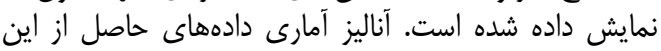

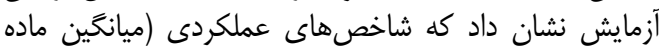

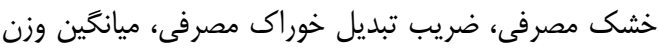

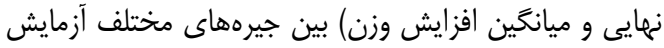

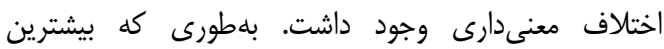

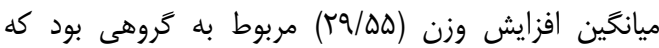

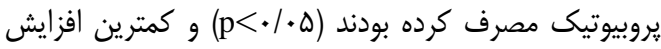

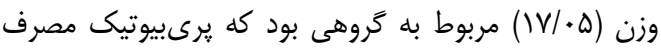

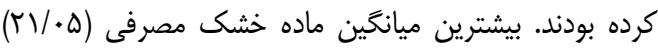

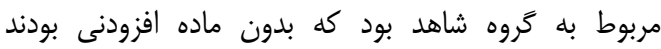

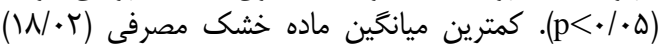

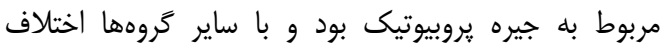

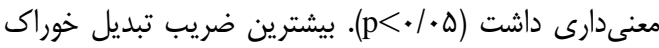

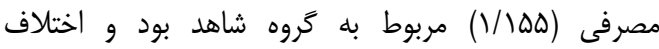

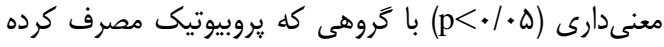

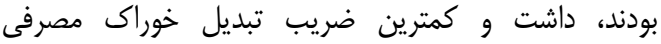

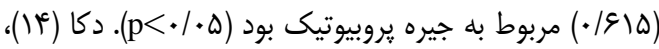


جدول ب- اثر جيرههاى آزمايشى بر شاخصهاى عملكردى گوسالههاى هلشتاين

Table 3. Effects of ingredients diets on functional parameters of Holstein calves

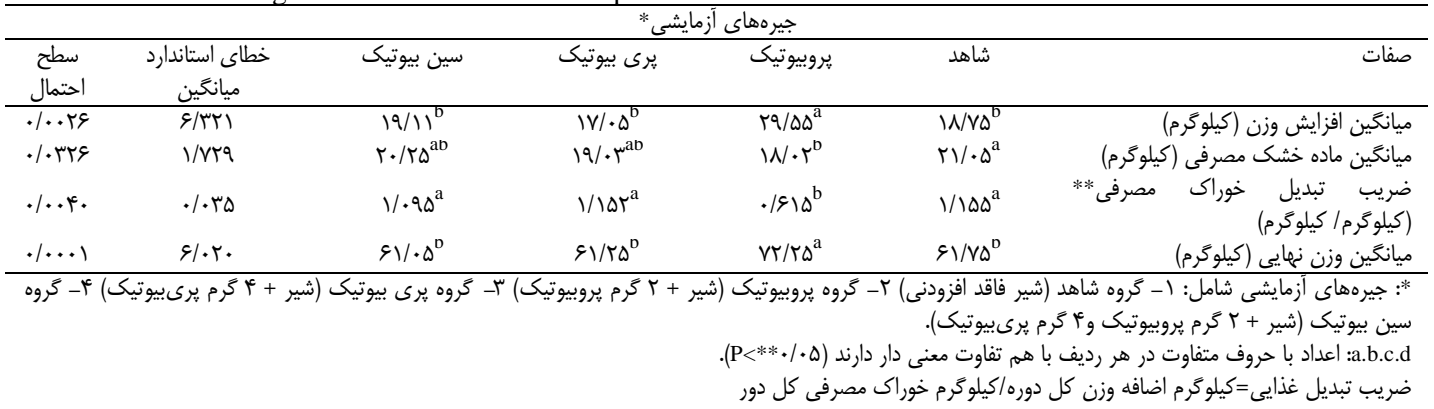

آزمايشات تعداد تكرارهاى بيش از كار أس رأس توصيه شده است (DV،IT)

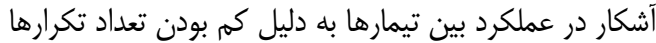

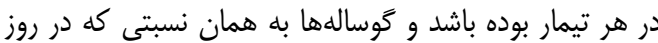

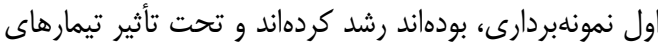

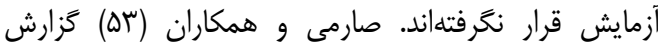

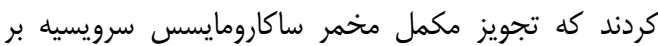
روى شاخصهاى رشد اسكلتى و كارايى اثر مارون معنىدارى نداشت.

\section{رشد اسكلتى در سنين مختلف}

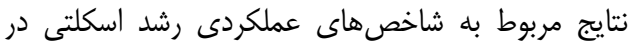

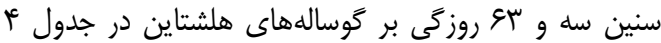

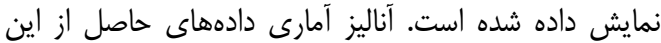

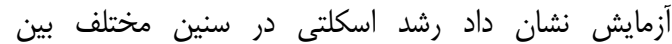

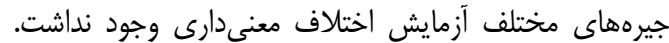

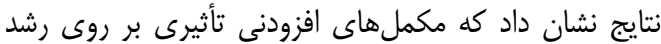

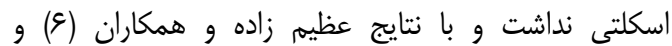

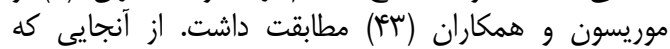

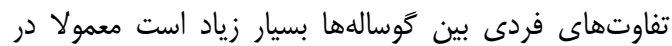

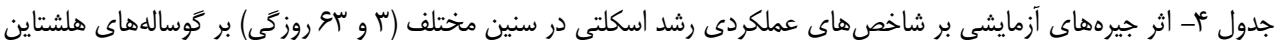
Table 4. Effects of experimental diets on the functional parameters of skeletal growth at different ages (3 and 63

\begin{tabular}{|c|c|c|c|c|c|c|}
\hline \multicolumn{7}{|c|}{ جيرههاى أزمايشى * } \\
\hline سطح احتمال & خطاى استاندارد ميانگين & سين بيوتيك & يرى بيوتيك & يروبيوتيك & شاهد & صفات \\
\hline & & & & & & عرض لكن (سانتىمتر) \\
\hline 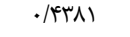 &.$/ 111$ & IV/VE & $M / \cdot 1$ & $|N| \cdot 1$ & IV/VE & كا روزگى \\
\hline ./rat & . & ro/vg & $r \Delta / r q$ & $r \Delta / v 1$ & $T \Delta / T$. & ارتفاع جدوزى (ساه (سانتىمتر) \\
\hline . & $\cdot / A V^{c}$ & $\mathrm{VQ} / \mathrm{AV}$ & $v a / 19$ & $\mathrm{VQ} / \mathrm{qV}$ & $V N / q$. & "ب روزگى \\
\hline •/OHYI & אזו/ & $\Lambda \mathrm{V} / \mathrm{\Lambda}$. & $\Lambda V / r q$ & $M / I F$ & $M / \Gamma \omega$ & سو روزگى \\
\hline . NQF. & . / R . & $\mid Q / 9 V$ & $10 / \wedge q$ & $\mid Q / \& D$ & $10 / \uparrow q$ & r روزي \\
\hline - /IFAT & . IMF & $19 / \%$. & $19 / 94$ & $19 / 9 \mathrm{~V}$ & $18 / \mu$. & سوروزگى \\
\hline
\end{tabular}

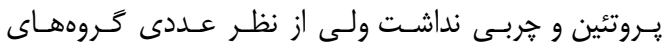

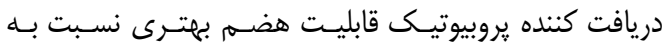

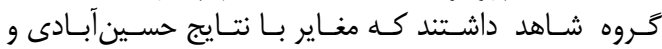

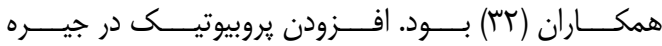

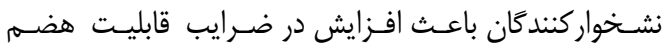

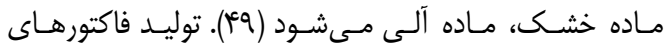

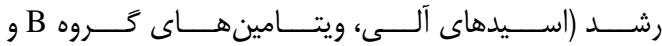

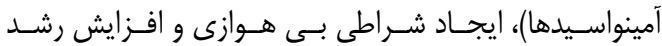

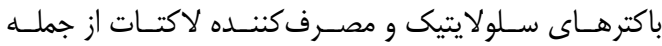

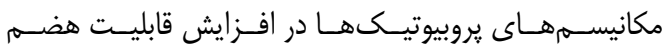

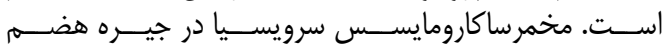

ضرايب قابليت هضم ظاهرى مواد مغذى

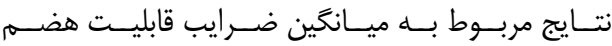

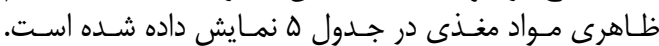

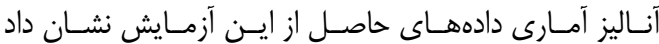

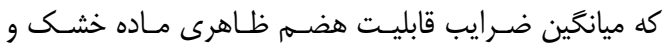

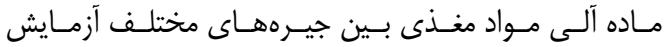

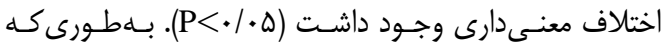

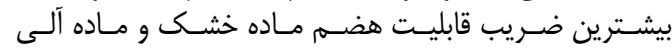

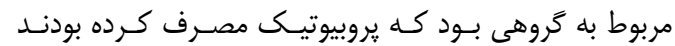

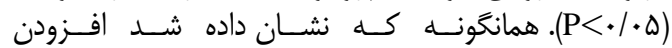

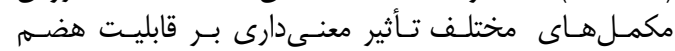




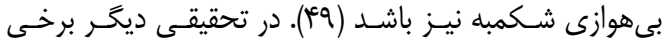

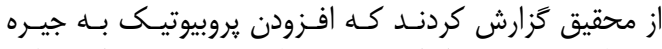

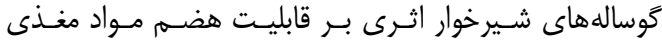

نداشت (rT).

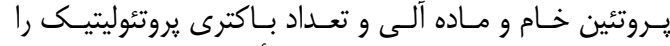

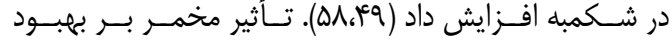

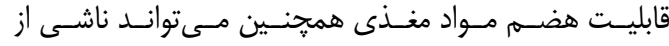

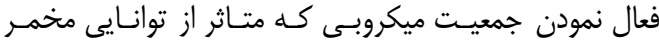

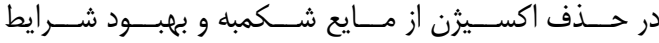

جدول هـ اثر جيرههاى آزمايشى بر ميانگين ضرايب قابليت هضم ظاهرى مواد مغذى (درصد) Table 5. Effects of experimental diets on the average apparent digestibility coefficient of nutrients (percentages)

\begin{tabular}{|c|c|c|c|c|c|c|}
\hline \multicolumn{7}{|c|}{ جيرههاى أزمايشى * } \\
\hline سطح احتمال & خطاى استانداردميانگين & سين بيوتيك & يرى بيوتيك & يروبيوتيك & شاهد & صفات \\
\hline $.1+\cdot 10$ & $r / \cdot r$. & $V Q / T D^{D}$ & $V V / r Q^{D}$ & $\Lambda F / r \Delta^{a}$ & $V \Lambda / .^{D}$ & ماده خشك \\
\hline - / Drq & $\Lambda / T \Delta$ & $V I / T \Delta$ & $V T / T \Delta$ & $V F / r \Delta$ & $V T / V Q$ & קربى \\
\hline.$/ 149$ & ه/१।द & $V \Psi / V \Delta$ & $V / T \Delta$ & $V \Delta / V \Delta$ & $V T / V D$ & يروتئين \\
\hline $.1 .+k t$ & $\Delta / V Y q$ & $V F / \Delta \cdot{ }^{D}$ & $\vee Q / \Delta \cdot{ }^{a b}$ & $\Lambda) / v \Delta^{a}$ & $\vee \& / .0^{D}$ & ماده آلى \\
\hline
\end{tabular}

سلامت دام شد (أ). تغييرات ناتمانى در تغذيـه حيوانـات و يـا

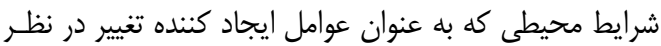

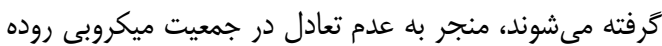

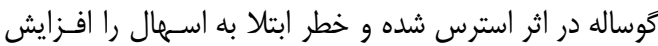

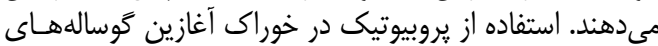

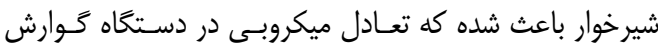

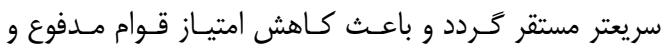

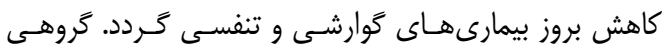

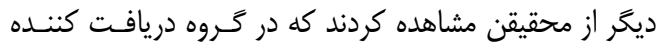

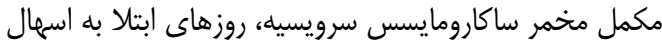

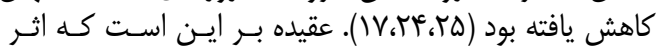

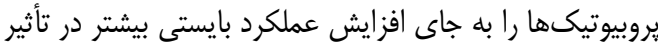
سودمندشان روى سلامتى دام ارزيابى كرد (عَ).
شاخصهاى سلامتى، قوام و سياليت مدفوع

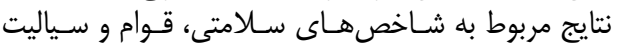

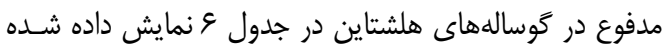

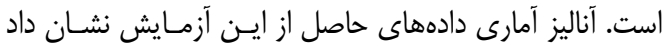

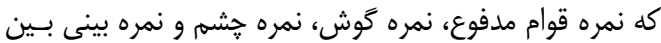

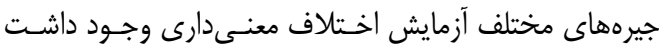

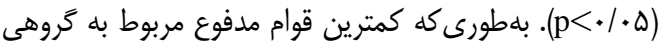

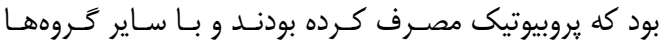

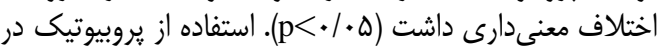

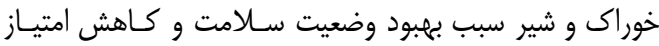

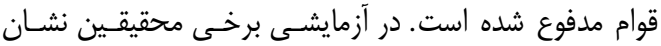

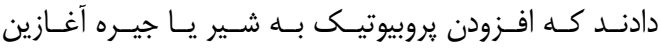

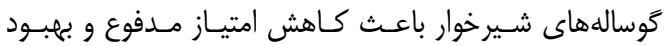

جدول عـ اثر جيرههاى آزمايشى بر شاخص هاى سلامتى، قوام و سياليت مدفوع در گوسالههاى هلشتاين Table 6. Effect of experimental diets on health indices, consistency and fluidity of stool in Holstein calves

\begin{tabular}{|c|c|c|c|c|c|c|}
\hline \multicolumn{7}{|c|}{ جيرههاى آزمايشى * } \\
\hline سطح احتمال & خطاى استانداردميانكين & سين بيوتيك & يرى بيوتيك & يروبيوتيك & شاهد & صفات \\
\hline $.1 .+94$ &.$/ .94$ & $1 / 9 \Delta \mu^{a}$ & $1 / \Lambda \Delta \cdot^{a}$ & $1 / \Delta T F^{D}$ & $1 / 19 \cdot^{a}$ & قوام مدفوع \\
\hline .1 .999 & $.1 \cdot \mathrm{Vq}$ & $1 / \Delta \cdot V$ & $1 / \Delta 9$. & $1 / \cdot v r$ & 1/RTy & سياليت مدفوع \\
\hline 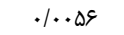 & . & $1 / \backslash \Delta V^{\mathrm{ad}}$ & $1 / 1 V \Delta^{a}$ & $1 / \cdot 10^{\mathrm{D}}$ & $1 / 11 v^{\mathrm{av}}$ & نمره كوش \\
\hline .1 .1 .9 & .1 .11 & $1 / \cdot A v^{\mathrm{av}}$ & $1 / r \ldots{ }^{a}$ & $1 / \cdot 1 \cdot^{0}$ & $1 / \cdot \Delta \Delta^{D}$ & نمره بينى \\
\hline . /TQQ. & $.1 \cdot 11$ &.$/ 814$ & . DQST & $\cdot / \digamma \Delta \Delta \Delta$ & $\cdot / \Delta 1 D$ & تعداد روزهاى اسهال \\
\hline 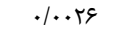 & . . . rav & $1 / \pi T r^{a D}$ & $1 / 11 r^{a}$ & $1 / \cdot 10^{\mathrm{D}}$ & $1 / F \Delta r^{\mathrm{a}}$ & نمره حشم \\
\hline
\end{tabular}


1. Abe, F. N. Ishibashi and S. Shimamura 1995. Effect of administration of bifidobacteria and lactic acid bacteria to newborn calves and piglets. Journal of Dairy Science, 78: 2838-2846.

2. Afshar Mazandaran, N.V. and A. Rajab. 2002. Probiotics and their application in feeding livestock and poultry. Nourbakhsh Publication, (In Persian).

3. Agarwal, N., D.N. Kamra, L.C. Chaudhary, A. Sahoo and N.N. Pathak. 2002. Microbial status and rumen enzyme profile of crossbred calves fed on different microbial feed additives. Letters in Applied Microbiology, 34: 329-36.

4. Aldana, C., S. Cabra, A. Carlos, F. Carvajal and F. Rodriguez. 2009. Effect of probiotic compound in rumen development, diarrhea incidence and weight gain in young Holstein calves. World Academy of Science, Engineering and Technology, $33 \mathrm{pp}$.

5. Ali, M.F., B.E. Ei-Saidy, M.I. Mohsen and M.M.E. Kalalfalla. 2005. Performance of lambs fed on ration containing soybean meal treated with formaldehyde and probiotics: Productive and eproductive performance. Egyptian Journal of Nutrition and Feeds, 8: 511-527.

6. Azimzadeh, A., A. Asadi al-Mutati, A. Akbar Khadem and J. Mohammad Moradi. 2015. Effects of feeding a synbiotic additive on the growth and health performance of Holstein calves. Animal production research. Sixth year Number, 12: 113-105 (In Persian).

7. Ballou, M.A. 2011. Case study: Effects of a blend of prebiotics, probiotics, and hyperimmune dried egg protein on the performance, health and innate immune responses of Holstein calves. The Professional Animal Scientist, 27: 262-268.

8. Baron, E.J. and S.M. Finegold. 1990. Diagnostic Microbiology. $8^{\text {th }}$ ed. the CV. Mosby Company. Torento, Canada.

9. Bayat Koharsar, J., A. Tahmasebi, A. Nasserian and M.R. Rezaei. 2014. Effect of using probiotic produced in laboratory on the performance of infant calves. 6th Iranian Congress of Animal Sciences. Tabriz University, (In Persian).

10. Beauchemin, K.A., W.Z. Yung, D.P. Morgavi, G.R. Ghorbani and J.A.Z. leedle. 2003. Effects of bacterial direct-fed microbial and yeast on site and extent of digestion, blood chemistry and ubclinical ruminal acidosis in feedlot cattle. Journal of Animal Science, 81: 1628-1640.

11. Chimwano, A.M., E.R. Orskov and C.S. Stewart. 1976. Effect of dietary proportions of roughage and concentrate on rate of dried grass disappearance in the rumen of sheep. Proceedings of the Nutrition Society, 35(2): 101A-102A.

12. Cruywagen, C.W., I. Jordaan and L. Venter. 1996. Effect of Lactobacillus acidophilus supplementation of milk replacer on preweaning performance of calves. Journal of Dairy Science, 79: 483-486.

13. Darreh Zarashkipour, M., Kh. Parsaei Mehr, F. Hossein Zadeh and P. Farhomand. 2013. The effect of different levels of prebiotic supplementation (E-max) on digestibility and some biochemical parameters of serum of West Azarbaijan native pups. Veterinary Clinic Pathology, 7(2): 321-314, (In Persian).

14. Deka, R.S. 2009. Effect of probiotic Biobloom as growth promoter in kids. Indian Veterinary, 86(11): 1192-1193.

15. Dvorak, R.A., K.A. Jacques and K.E. Newman. 1998. Mannan oligosaccharide, fructooligosaccharide and Carbadox for pigs 0-21 dayspost-weaning. Journal of Animal Science, 76(2): 64-76.

16. Enjalbert, F., J.E. Garrett, R. Moncoulon, C. Bajourthe and P. Chicoteau. 1999. Effects of yeast culture (saccharomyces cerevisiae) on ruminal digestion in non-lactating dairy cows. Animal Feed Science and Tech, 183: 140-151.

17. Erb, O. 1992. Prevention of diarrhoea in the calf with live yeast. Zur Durchfallprophylaxe mit lebender Hefe beim Kalb, pp:109.

18. Firouznia, H. 2013. Effect of Saccharomyces cerevisiae on the production, composition of milk and blood parameters in Holstein lactating cows. master thesis. Faculty of Agriculture, Tabriz University, (In Persian).

19. Fowler, J., R. Kakani, A. Haq, Ja. Byrd and Ca. Bailey. 2015. Growth promoting effects of prebiotic yeast cell wall products in starter broilers under an immune stress and clostridium perfringens challenge. The Journal of Applied Poultry Research, 24: 66-72.

20. Frizzo, L.S., M.V. Zbruna, L.P. Sotoa and M.L. Signorinib. 2011. Effects of probiotics on growth performance in young calves: a meta-analysis of randomized controlled trials. Animal Feed Science and Technology, 169: 147-156.

21. Fujiwara, K., M. Yamazaki, H. Abe, K. Nakashima, Y. Yakabe, M. Otsuka, Y. Ohbayashi, Y. Kato, K. Namai, A. Toyoda, Y. Miyaguchi and Y. Nakamura. 2009. Effect of Bacillus subtilis var. natto fermented soybean on growth performance, microbial activity in the caeca and cytokine gene expression of domestic meat type chickens. The Journal of Poultry Science, 46: 116-122.

22. Fuller, R. 1992. Probiotics: the scientific basis chapman and Hall.London.pp:1-20Galip N, 2006. Effects ofdietary Saccharomyces cerevisiae live Yeast culture supplementation on ruminal digestion and protozoa count in rams fed with diets with or high ratio forage / concentrate.Faculty of veterinarymedicine. 16059 bursa /Turkey, 157(12): 609-613.

23. Fuller, R. 1977. The importance of lactobacilli in maintaining normal microbial balance in the crop. British Poultry Science, 18: 85-94.

24. Fleige, S.W., P. Binger, H.H.D. Meyer and M.W. Pfaffl. 2007. Effect of lactulose on growth performance and intestinal morphology of preruminant calves using a milk replacer containing Enterococcus faecium. The Animal Consortium, 1: 367-373. 
25. Galvao, K.N., J.E. Santos, A. Coscioni, M. Villasenor, W.M. Sischo and A.C. Berge. 2005. Effect of feeding live yeast products to calves with failure of passive transfer on performance and patterns of antibiotic resistance in fecal Echerchia coli. Reproduction Nutrition Development, 45: 427-440.

26. Gibson, G.R. 2004. Fibre and effects on probiotics (the prebiotic concept). Clinical Nutrition Supplements, 1(2): 25-31.

27. Gibson, G.R. and M.B. Roberfroid. 1995. Dietary modulation of the human colonicmicrobia: Introducing the concept of prebiotics. Journal of Nutrition, 125: 1401-1412.

28. Heinrichs, A.J., C.M. Jones and B.S. Heinrichs. 2003. Effects of mannan oligosaccharide or antibiotics in neonatal diets on health and growth of dairy calves. Journal of Dairy Science, 86: 4064.

29. Heydari Khormizy, S., R, Dehghan, M, Benadiki, K. Researcher and A. Zali. 2007. Study of the effect of probiotic and fungal probiotics on production performance of Holstein cattle in early lactation. Master's thesis, University of Tehran, (In Persian).

30. Higginbotham, G.E. and D.L. Bath. 1993. Evaluation of Lactobacillus fermentation cultures in calf feeding systems. Journal of Dairy Science, 76: 615-620.

31. Hossain, S.A., S. Parnerkar, N. Haque, R.S. Gupta, D. Kumar and A.K. Tyagi. 2012. Influence of dietary supplementation of live yeast (Saccharomyces cerevisiae) on nutrient utilization ruminal and biochemical profiles of Kankrej calves. International Journal of Applied Animal Research, 1(1): 3038.

32. Hossein Abadi, M., M. Dehghan Banadaki and A. Zali. 2013. Effect of adding probiotic bacteria in milk or initial feed on growth performance, health condition, blood and stomatal parameters of Holstein calves. Animal production research. forth year. Number, 8: 69-57 (In Persian).

33. Houdijk, J.G.M., M.W. Bosch, S. Tamminga, M.W.A. Verstegen and E.B. Berenpas. 1999. Apparent ileal and total-tract nutrient digestion by pigs as affected by dietary non-digestible oligosaccharides. Journal of Animal Science, 77: 148-158.

34. Nisbet, D.J. and S.A Martin. 1991. Effect of Saccharomyces cerevisiae culture on lactate utilization by the ruminal bacterium Selenomonas ruminantium. Journal of Animal Sciences 69:4628-4633

35. Kogan, G. and A. Kocher. 2007. Role of yeast cell wall polysaccharides in pig nutrition and health protection. Livest Science, 109-165.

36. Kong, X.F., G.Y. Wu and Y.L. Yin. 2011. Roles of phytochemicals in amino acid nutrition. Front. . Biological Sciences, S3: 372-384.

37. Krehbiel, C.R., S.R. Rust, G. Zhang and S.E. Gilliland. 2003. Bacterial direct-fed microbials in ruminant diets: Performanceresponse and mode of action. Journal of Animal Science, 81: E120-E132.

38. Kritas, S.K. and R.B. Morrison. 2005. Evaluation of probiotics as a substitute for antibiotics in a large pig nursery. The Veterinary Record, 156: 447-448.

39. Kung, L., E.M. Kreck, R.S. Tung, A.O. Hession, A.C. Sheperd, M.A. Cohen, H.E. Swain and J.A.Z. Leedle. 1997. Effects of a live yeast culture and enzymes on in-vitro ruminal fermentation and milk production of dairy cows. Journal of Dairy Science, 80: 2045-2057.

40. Mainardi, S.R., B.A. Hengst, S.G. Nebzydoski, L.M. Nemec and T.F. Gressly. 2012. Effects of obomasal oligofroctose on blood and feces of Holstein steers. Department of Animal and Food science, 45: 155-161.

41. Mohamadi Roodposhti, P. and N. Dabiri. 2012. Effects of probiotic and prebiotic on average daily gain, fecal shedding of Escherichia Coli and immune system status in newborn female calves. AsianAust. Journal of Animal Science, 9: 1255-1261.

42. Mohammadi, G.R., M. Mori, F. Hamidi and M. Ghavami. 2004. Feild trial evaluation of kolbin RC (Rotavirus,Coronavirus/Escherichia coli) vaccine for prevention of neonatal calf diarrhea in dairy herd.11th international conference of the association of institutions for tropical veterinary medicine and 16th veterinary association malaysia congress 23-27 August,2004 ,Malaysia, 270-272.

43. Morrison, S.J., S. Dawson and A.F. Carson. 2010. The effects of mannan oligosaccharide and Streptococcus faecium addition to milk replacer on calf health and performance. Livestock Science, 131: 292-296.

44. Mwenya, B., B. Sntoso, C. Pen, R. Morikava, K. Takaura and K. Umetsu. 2005. Effects of yeast Culture and galacto-oligosaccharides on luminal fermentation in Holstein cows. Journal of Dairy Science, 88: 1404-1412.

45. Nakanishi, Y., C.W. Arave and P.H. Stewart. 1993. Effect of feeding Lactobacillus acidophilus yogurt on performance and behavior of dairy calves. Journal of Dairy Science, 76(1): $244 \mathrm{pp}$.

46. Novak, K.N., E. Davis, C.A. Wehnes, D.R. Shields, J.A. Coalson, A.H. Smith and T.G. Rehberger. 2012. Effect of supplementation with an electrolyte containing a Bacillus-based direct-fed microbial on immune development in dairy calves. Research in Veterinary Science, 92: 427-434.

47. Pieper, R., P. Janczyk, V. Urubschurov, U. Korn, B. Pieper and W.B. Souffrant. 2009. Effect of a single oral administration of Lactobacillus plantarum DSMZ 8862/8866 before and at the time point of weaning on intestinal microbial communities in piglets. International Journal of Food Microbiology, 130: 227-232.

48. Piras, C. and S. Bovolenta. 1995. The use of brewer's yeast (Saccharomyces cerevisiae) for weaning calves. Zootecnica e Nutrizione Animale, 21: 57-61.

49. Plata, F.P., G.D. Mendoza, J.R. Blrcena-Gama and S. Gonzalez. 1994. Effect of a Yeast culture (Saccharomyces Cerevisia) on neutral detergent fiber digestion in steers fed oat straw based diets. Animal Feed Science, 4: 203-210.

50. Rameshwar S., L.C. Chaudhary, D.N. Kamra and N.N. Pathak. 1998. Effect of dietary supplementation with yeast cell suspension (Saccharomyces cerevisiae) on nutrient utilisation and growth response in crossbred calves. Asian-Australasian Journal of Animal Sciences, 11: 268-271. 
51. Riddell, J.B., A.J. Gallegos, D.L. Harmon and K.R. Mcleod. 2010. Addition of a Bacillus based probiotic to the diet of pre ruminant calves: influence on growth, health and blood parameters. Intern. Journal of Applied Research in Veterinary Medicine, 8: 78-85.

52. Santoso, B., B. Mwenya, C. Sar, Y. Gamo, T. Kobayashi and R. Morikawa. 2004. Effects of supplementing galacto-oligosaccharids, Yucca schidigra or nisin on rumen metanogenesis, nitrogen and energy metabolism in sheep. Livestock Production Science, 91: 209-217.

53. Saremi, B., A.A. Naserian, M. Bannayan, and F. Shahriary. 2004. Effect of yeast (Saccharomyces cerevisiae) on rumen bacterial population and performance of Holstein female calves. Agricultural Sciences and Technology, 18: 91-103.

54. SAS, Institute. 2003. SAS User's Guide. Version 9.1 ed. SAS Institute Inc., Cary, NC.

55. Savage, D.C. 1987. Microorganisms associated with epithelial surfaces and the stability of the indigenous gastrointestinal microflora. Nahrung, 31(5-6): 383-395.

56. Taylor, D.J. 2001. Effects of antimicrobials and their alternative. British Journal of Poultry Science, 42: 67.

57. Timmerman, H.M., L. Mulder, H. Everts, D.C. van Espen, E. van der Wal, G. Klaassen, S.M.G. Rouwers, R.F. Hartemink, M. Rombouts and A.C. Beynen. 2005. Health and growth of veal calves fed milk replacers with or without probiotics Journal of Dairy Science, 88: 2154-2165.

58. Wallace R.J. 1994. Ruminal microbiology, biotechnology and ruminant nutrition: progress and problems. Journal of Dairy Science, 72: 2992-3003. 


\title{
Effects of Probiotic and Peribiotic Supplementation in Milk on Performance and Nutrition Digestibility in Holstein Calves
}

\author{
Masood Didarkhah ${ }^{1}$ and Moslem Bashtani ${ }^{2}$ \\ 1- Assistant Professor, Faculty of Agriculture Sarayan, University of Birjand \\ (Corresponding Author: masooddidarkhah@birjand.ac.ir) \\ 2- Professor, Department of Animal Sciences, Faculty of Agriculture, University of Birjand \\ Received: December 9, 2017 \\ Accepted: May 16, 2018
}

\begin{abstract}
The purpose of this study was to evaluate the effects of feeding probiotic and peribiotic supplements on milk yield, feed intake, fecal consistency and digestibility of Holstein calves. Treatments included: 1- control group (no additive milk) 2- probiotic group (milk +2 gr probiotic) 3-prebiotic group (milk +4 gr peribiotic) 4 - synbiotic group (milk +2 gr probiotic and $4 \mathrm{gr}$ perbiotic). Calves were weighed at the age of 3, 30 and 63 days after milking and feed intake weight was measured and recorded for every calves from the age of 10 days to the end of the period. For diarrhea, feces were observed weekly and feces score and fluidity were examined. At the end of the experiment (7 final days), the total stools of livestock were separately collected and weighed $20 \%$ sample was taken to investigate digestibility of nutrients. The chemical composition of fecal specimens and experimental diets including dry matter, fat, organic matter and protein were determined according to the AOAC method. The results of the experiment showed that the highest mean weight in the age of 30 and 63 days was related to the calves that used probiotics and had a significant difference with other groups. The lowest mean weight at the age of 63 was related to the synbiotic diet. The worst conversion ratio was in the control group and had a significant difference with the group that consumed probiotics. The best feed conversion ratio was probiotic diet and had a significant difference with other groups ( $P$ $<0.05)$. The highest digestibility of dry matter and organic matter belonged to the group that consumed probiotics and had a significant difference with other groups. The lowest fecal consistency was in the group that consumed probiotics and had a significant difference with other groups $(\mathrm{P}<0.05)$. The lowest stool consistency was related to the group that used probiotics and had a significant difference with other groups $(\mathrm{P}<0.05)$. In general, the results showed that the use of probiotic had a significant effect on the performance, health and digestibility of Holstein calves.
\end{abstract}

Keywords: Peribiotics, Probiotics, Ruminants 